\title{
Non-heart-beating organ donation
}

Transplantation programmes throughout the world are struggling to maintain current levels of organ donation. Transplant waiting lists continue to rise and the resulting deficit is growing year on year.

Cadaveric heart-beating donation (HBD), where death is confirmed after brain stem testing, still provides the majority of organs. Over the past $10 \mathrm{yr}$ this type of donation has fallen by approximately $20 \%$. Efforts are being made to reverse this trend and to explore alternative sources for donation.

Non-heart-beating organ donation (NHBOD), where death is confirmed after irreversible cardiopulmonary arrest, was widely practised until the early 1970s. It was abandoned when brain stem testing was introduced to establish death, leading to vastly improved results from these donors. Advancements in immunosuppression and surgical techniques now mean that the prognosis for organs retrieved from NHBOD is the same, or in certain cases improved, compared to HBD. Consequently, NHBOD can no longer be dismissed on the grounds of organ viability. At present NHBOD contributes only 3\% of transplanted organs, mainly because centres are not undertaking the procedure. For example, there are only four sites in the UK currently practising NHBOD. Figures from these centres [1] suggest that NHBOD could increase organ availability by up to $20 \%$ and its introduction would therefore make a major contribution to reducing the waiting lists.

There are key differences between HBD and NHBOD. The implementation of NHBOD would involve changes to the existing transplant framework. As anaesthetists or intensivists, we are essential members of this framework. Our contribution to the heart-beating donor programme includes the identification of potential donors in general and neurosurgical intensive care units (ICUs), testing brain stem function and documenting death. We also counsel relatives and obtain consent, notify the transplant co-ordinator and retrieval team, transfer the patient to

Correspondence to: Nick Pace, Department of Anaesthesia, Western Infirmary, Glasgow, UK. E-mail: nick.pace@northglasgow.scot.nhs.uk; Tel: +44 (0)141 2112069

Accepted for publication July 2003 EJA 1470 the operating room and provide perioperative care. What can we expect our role to be in NHBOD and what can we contribute to developing this resource?

NHBOD is the retrieval of tissue and solid organs from a body following irreversible cardiopulmonary arrest. Since the circulation is not restored for retrieval (although some centres reintroduce cardiac massage and lung ventilation to improve organ viability and buy time), the priority is to expedite removal of the organs before they deteriorate and transplantation cannot take place. Thus the clock is ticking from the moment of death.

There are a number of potential sources of nonheart-beating donors. The circumstances of their presentation will dictate our involvement as anaesthetists or intensivists. The Maastricht group has identified four well-established categories [2]:

Category 1: Dead on arrival at hospital. This group includes any individual who suffers an out-of-hospital arrest and arrives in hospital with an accurate documentation of the time of death and pre-hospital resuscitation.

Category 2: Unsuccessful resuscitation. This includes any patients who arrest in hospital and again have a failed resuscitation accurately documented.

Category 3: Awaiting cardiac arrest. These are patients presenting to the Accident and Emergency (A\&E) department with a non-survivable injury or patients, in general or neurosurgical intensive therapy units (ITUs), whose death is inevitable. They do not fulfil brain stem death criteria and therefore cannot become a heart-beating donor.

Category 4: Cardiac arrest in a beart-beating donor. This refers to a patient whose death has been confirmed by brain stem testing but arrests before organ retrieval can take place.

An additional category has recently been suggested to identify a more specific group [3]:

Category 5: Unexpected cardiac arrest in a patient in ITU.

Categories 1, 2, 4 and 5 are described as 'uncontrolled' donation. This is because all of the preparations for retrieval have to take place from the time of death and be completed before the recommended 
warm ischaemic time of $45 \mathrm{~min}$ has been reached. These preparations include addressing consent, counselling relatives, preparation of the donor (tissue typing and viral status), mobilizing a surgical team and finally cold perfusion of the organs.

The anaesthetist present with the arrest team in the ward, on stand-by in the A\&E department - or, more significantly, at an unexpected cardiac arrest of one of our own patients in the ITU - could be involved in the declaration of death and notification of the transplant team.

Category 3 is a controlled donation. (Category 4 can occasionally be considered a controlled donation. This depends on how far along the process events have progressed when the patient arrests.) These patients are generally more stable, their condition is deemed to be futile and a decision has been made either not to introduce care or to withdraw care thereby making the time of death more predictable. The preparations mentioned above can be made before death occurs and the retrieval can be scheduled. This makes it easier to remain within the recommended ischaemic time and consequently the organs procured should be of a higher quality and carry a better prognosis after transplantation.

The advantage of a less time-pressured process compared to uncontrolled donation means that controlled donation offers the best opportunity to introduce NHBOD successfully. It has been proposed that general and neurosurgical intensive care should therefore become the focus for identifying potential donors.

The introduction of controlled donation is not straightforward. There are concerns regarding several ethical and legal issues specific to this type of organ retrieval. If these are not addressed properly there will almost certainly be a loss of public confidence with a negative effect on the overall rates of donation.

The identification of potential donors presents a problem for the anaesthetist. The judgement that a patient's condition is futile, the process of withdrawing care and the provision of palliative measures to allow a comfortable death are well established in intensive care. However, there is concern that the timing of this process could be influenced by the need to ensure optimum organ viability if considering NHBOD. To avoid criticism of a conflict of interest, the initial decision to withdraw care should be made on the basis of multi-disciplinary discussions, which include the intensivist, parent consultant, senior nursing staff and the next-of-kin. It should precede and be clearly separate from consideration for donation, and should not involve any of the transplant team. The process itself needs to be transparent and meticulously documented.

Introducing the subject of withdrawing treatment is a difficult time for the family. Involving the family in the decision-making process will help to prevent mistrust and resentment. It is important to avoid upsetting the relationship that will have developed between the family and the medical staff through the patient's time in intensive care. This relationship will help the medical and nursing staff to support the family through their bereavement and the process of donation.

Obtaining consent for NHBOD can be awkward; however, our continuing care commitment to the patient and their family should put us in the best position to do this sensitively. Consent should therefore be the remit of the intensivist and not delegated to a member of the transplant team. They must give permission to obtain samples for virology, blood grouping and human leucocytes antigen typing. They should have the process explained to them and made aware that there will be limited time to spend with their relative after death due to the need to expedite retrieval. They should also understand that the solid organs may no longer be viable by the time they are retrieved and therefore of no use for transplantation. The family should understand that they could withdraw consent at any time.

Organ viability is not only dependent upon the patient's pre-mortem condition and the ischaemic time until retrieval. The withdrawal of supportive care can adversely affect the proposed donor organs if it results in a prolonged deterioration in physiological variables before cardiopulmonary arrest. A trial of withdrawal of care would help to identify those patients who would progress rapidly to cardiopulmonary arrest and thereby more likely to provide viable organs. However, we continue to have a duty of care to the potential non-heart-beating donor until the moment of death. This prevents any intervention, which is not in the best interests of the patient or may accelerate their death. A trial withdrawal could be seen to fulfil the best interests of the patient if these have been expressed as the donation of viable organs. If this trial suggests a prolonged deterioration in the patient and therefore a poor prognosis for donation, then we could potentially avoid subjecting the patient and their family to an unnecessary procedure.

The administration of corticosteroids, antibiotics and heparin has been employed to try to minimize organ deterioration after death. These should be given before the withdrawal of care to have a beneficial effect. This can again be justified on the basis that they may help to fulfil the patient's wish to donate viable organs. However, they should not be given if there is any contraindication that might accelerate the patient's death, e.g. risk of catastrophic haemorrhage with heparin. The potential benefits of these treatments should be explained when NHBOD is discussed and included in the same process of achieving consent. At present there is no clinical evidence 
to support their use and the implementation of any of these treatments is dependent on local policy. The intensivist should be responsible for administering any agreed treatment to the patient in intensive care and we therefore need to be involved in establishing such a policy.

Aorto-femoral cannulation and perfusion of the solid organs with a cold preservative is another method, more widely used, to maintain organ viability. This is usually performed via a femoral cut-down after the declaration of death. It helps to reduce the warm ischaemic damage to the organs thereby buying time to allow a more relaxed surgical retrieval, the maximum recommended time for retrieval increases from $45 \mathrm{~min}$ to $2 \mathrm{~h}$. Timing is again crucial as early intervention after death produces the best results. The advantage of controlled donation is that the time for retrieval is less pressured and it would seem appropriate that if cannulation was felt to be necessary it should be reserved until the patient arrives in the operating room. This would avoid exposing the family and the continuing care staff to an intrusive procedure at such a sensitive time. If it is in practice to use cannulation then it should be described when obtaining consent for NHBOD from the patient or family.

The advantages of cannulation are more applicable to uncontrolled donation. The extra time provided by cold perfusion facilitates completion of the retrieval process within the recommended ischaemic times. Uncontrolled donation is characterized by having to make all preparations, including obtaining consent, after the patient has been declared dead. Donation has therefore not been discussed with the patient and often the next-of-kin are not present. This raises a problem concerning consent. It is essential that cannulation is not felt to be an assault on the patient and that it does not cause distress to the family. If it can be established that the patient expressed a wish to donate organs during life, either by carrying a donor card or having registered on the donor list, then it would be reasonable to proceed with cannulation. Informed consent could then be sought from the family. The family may, however, refuse consent and then the procedure should be abandoned. It is important to respect the opinion of the family even if this is at the expense of the final wishes of the deceased. Ignoring them would cause upset and distress and have a negative impact on public confidence in the transplantation programme, thereby possibly affecting overall rates of donation. If the patient's wishes cannot be established then consent should be obtained from the family before cannulation and if this is not possible then cannulation should not be attempted. Most jurisdictions carry a legal requirement to seek the opinion of the patient's next-of-kin and retrieval, including cannulation, should not proceed without their consent.
The intensivist is responsible for managing the withdrawal of supportive care. Ideally this should result in the patient progressing rapidly to cardiopulmonary arrest with the minimum of discomfort and distress. Clinical experience or the result of a trial withdrawal, or both, will help to determine the most appropriate level for withdrawing care and hopefully produce an outcome that facilitates NHBOD. The amount of distress and discomfort produced should be anticipated and both sedation and analgesia titrated accordingly. It is essential that the patient is comfortable. This helps to minimize the psychological trauma in the family and the staff from witnessing the events surrounding the patient's death.

The intensivist should certify the patient's death. This should occur in the ICU without interference from the transplant team and thus seen to be separate from the process of retrieval. There must be confidence that death is final and absolute to ensure that there is no risk of awareness or suffering during retrieval. Unfortunately there is some confusion regarding the legal definition of death, i.e. whether it is cardiorespiratory cessation or brain death. The two are not identical. Different jurisdictions or NHBOD protocols use either definition or in some cases both. However, some (e.g. Barcelona and Tokyo) are vague as to exactly how death is to be determined and the point at which organ preservation or removal can be undertaken.

In practice the patient is accepted to have died if not hypothermic and irreversibly unresponsive, apnoeic and asystolic. However, 'irreversible' is difficult to define. Although cardiopulmonary arrest results in a patient that is unresponsive, apnoeic and asystolic the patient can still recover either spontaneously or through resuscitation. The moment of arrest is therefore not irreversible and therefore cannot be used to declare death to allow organ procurement. The problem is how much time must pass after the arrest before death becomes irreversible. 'Auto-resuscitation' or the spontaneous recovery of a circulation has only ever been reported within 2 min of arrest. This would appear to be the minimum amount of time that should pass for certification to be acceptable. Resuscitative measures could restore the circulation and brain function after a patient has been unresponsive, apnoeic and asystolic for up to $10 \mathrm{~min}$. However, there would be a degree of neurological deficit proportionate to the duration and degree of hypoxia and hypoperfusion. The less effective resuscitation attempts are at minimizing physiological decline, the more rapidly the patient's state will become irretrievable. Without resuscitation, apnoea and asystole can be taken as irreversible after $2 \mathrm{~min}$. However, there is concern that permanent loss of brain function, even in the absence of resuscitation, may take longer than $2 \mathrm{~min}$. At present there is no method to determine 
when loss of brain function is irreversible. It is therefore appropriate to wait for a period of time that would put this beyond doubt and importantly maintain public and professional confidence in the process. The times used in practice range from $2 \mathrm{~min}$ at the University of Pittsburgh to up to $10 \mathrm{~min}$ in the original recommendation from the Maastricht group. This would have to be discussed and agreed at a local level before implementation.

Following the declaration of death, retrieval is performed and if viable the organs are subsequently transplanted. The body is then transferred to the mortuary where facilities should be provided for the family to visit. The relatives of the deceased may continue to have concerns and anxieties and it is important for them to believe that they have made a positive contribution by allowing donation. The intensivist should continue to have a role to play after the patient's death by offering counselling and helping the family through their bereavement.

NHBOD has the potential to increase available organ donation nationally by up to $17 \%$ in the UK but it is presently under-utilized. This is not a measure of its efficacy. Indeed, although the evidence to date has shown that there is a higher incidence of primary graft non-function and delayed graft function when comparing NHBOD to HBD, the 1 and $5 \mathrm{yr}$ survival of transplants in both groups is the same [4-6]. For a patient facing a lifetime of dialysis it is generally the long-term outcome that is important and NHBOD can make a significant contribution to their quality and duration of life.

We have described the role that the anaesthetist/ intensivist should expect to play in NHBOD, particularly with the development of controlled donation. The ethical and legal issues considered will influence our practice and it is essential that we are involved in their resolution at a local and national level. Early involvement of local authorities that deal with investigate deaths (e.g. police, coroners, and magistrates) is essential prior to the introduction of any protocol. This will give doctors and the public confidence in the process of NHBOD and ensure its success.

M. Steven, N. Pace

Department of Anaesthesia

Western Infirmary

Glasgow, $U K$

\section{Acknowledgement}

Dr N. Pace is a member of the Ethics Committee of the British Transplantation Society. The views expressed are his own.

\section{References}

1. UK Transplant, UK Transplant Activity Report. 2001. ISSN 1477 8505: 23.

2. Kootstra G, Daemen JH, Oomen AP. Categories of nonheart-beating donors. Transplant Proc 1995; 27: 2893-2894.

3. Sanchez-Fructuoso AI, Prats D, Torrente J, et al. Renal transplantation from non-heart beating donors: a promising alternative to enlarge the donor pool. J Am Soc Nephrol 2000; 11: 350-358.

4. Weber M, Dindo D, Demartines N, Ambuhl P, Clavien PA. Kidney transplantation from donors without a heartbeat. New Engl J Med 2002; 347: 248-255.

5. Cho YW, Terasaki PI, Cecka JM, Gjertson DW. Transplantation of kidneys from donors whose hearts have stopped beating. New Engl J Med 1998; 338: 221-225.

6. Nicholson ML, Metcalfe MS, White SA, et al. A comparison of the results of renal transplantation from non-heartbeating, conventional cadaveric, and living donors. Kidney Internat 2000; 58: 2585-2591.

\section{Further reading}

British Transplant Society: Guidelines for Non-heart Beating Organ Donation Scottish Transplant Group: Draft, January 2002.

DeVita MA, Snyder JV, Arnold RM, Siminoff LA. Observations of withdrawal of life-sustaining treatment from patients who became non-heart-beating organ donors. Crit Care Med 2000; 28: 1709-1712.

Ethics Committee, American College of Critical Care Medicine; Society of Critical Care Medicine. Recommendations for nonheartbeating organ donation. A position paper. Crit Care Med 2001; 29: 1826-1831.

Non-beart-Beating Organ Transplantation: Medical and Ethical Issues in Procurement. Institute of Medicine, Washington DC, USA: National Academy Press, 1997.

Truog RD, Cist AF, Brackett SE, et al. Recommendations for end-of-life care in the intensive care unit: The Ethics Committee of the Society of Critical Care Medicine. Crit Care Med 2001; 29: 2332-2348.

Whetstine L, Bowman K, Hawryluck L. Pro/con ethics debate: is nonheart-beating donation ethically acceptable? Crit Care 2002; 6: 192-195.

Apology: Greenwich Medical Media regret that due to a printers' error the spine of some copies of the October 2003 issue were incorrectly marked 'August' instead of 'October'. All other spine details are correct. 